\title{
Systematic Mapping Study on Software Engineering for Sustainability (SE4S)
}

\author{
Birgit Penzenstadler, \\ Ankita Raturi, \\ Debra Richardson \\ University of California, Irvine \\ \{bpenzens|araturi|djr\}@uci.edu \\ Henning Femmer \\ Technische Universität \\ München \\ femmer@in.tum.de
}

\author{
Coral Calero \\ Universidad de Castilla - La \\ Mancha, Spain \\ coral.calero@uclm.es
}

\author{
Xavier Franch \\ Universitat Politècnica \\ de Catalunya, Spain \\ franch@essi.upc.edu
}

\begin{abstract}
Background/Context: The objective of achieving higher sustainability in our lifestyles by information and communication technology has lead to a plethora of research activities in related fields. Consequently, Software Engineering for Sustainability (SE4S) has developed as an active area of research. Objective/Aim: Though SE4S gained much attention over the past few years and has resulted in a number of contributions, there is only one rigorous survey of the field. We follow up on this systematic mapping study from 2012 with a more in-depth overview of the status of research, as most work has been conducted in the last 4 years. Method: The applied method is a systematic mapping study through which we investigate which contributions were made, which knowledge areas are most explored, and which research type facets have been used, to distill a common understanding of the state-of-the-art in SE4S. Results: We contribute an overview of current research topics and trends, and their distribution according to the research type facet and the application domains. Furthermore, we aggregate the topics into clusters and list proposed and used methods, frameworks, and tools. Conclusion: The research map shows that impact currently is limited to few knowledge areas and there is need for a future roadmap to fill the gaps.
\end{abstract}

\section{Categories and Subject Descriptors}

D.2.1 [Software Engineering]: [Requirements, Methodologies]

\section{Keywords}

Sustainability, Software Engineering, Systematic Mapping Study

Permission to make digital or hard copies of all or part of this work for personal or classroom use is granted without fee provided that copies are not made or distributed for profit or commercial advantage and that copies bear this notice and the full citation on the first page. To copy otherwise, to republish, to post on servers or to redistribute to lists, requires prior specific permission and/or a fee.

EASE'14, May 13 - 14 2014, London, England, BC, United Kingdom Copyright 20XX ACM Copyright is held by the owner/author(s). Publication rights licensed to ACM. ACM 978-1-4503-2476-2/14/05...\$15.00, http://dx.doi.org/10.1145/2601248.2601256 ...\$15.00.

\section{MOTIVATION \& BACKGROUND}

Over the last decades, sustainability research has emerged as an interdisciplinary area; knowledge about how to achieve sustainable development has grown, while political action towards the goal is still in its infancy [1].

A sustainable world is broadly defined as "one in which humans can survive without jeopardizing the continued survival of future generations of humans in a healthy environment" 2]. This anthropocentric view of sustainability allows us to consider the implications of, and necessities for, human existence in the world.

Sustainability can be also discussed with reference to a concrete system - such as an ecological system, a human network, or a specific software system. Here, global sustainability implies the capacity for endurance given the functioning of all these systems in concert. Software Engineering for Sustainability has developed as a current focus of research as a result of software engineers engaging in issues regarding the impact of software systems on global sustainability.

Definition The term Sustainable Software can be interpreted in two ways: (1) the software code being sustainable, agnostic of purpose, or (2) the software purpose being to support sustainability goals, i.e. improving the sustainability of humankind on our planet. Ideally, both interpretations coincide in a software system that contributes to more sustainable living. Therefore, in our context, sustainable software is energy-efficient, minimizes the environmental impact of the processes it supports, and has a positive impact on social and/or economic sustainability (1 \& 2). These impacts can occur direct (energy), indirect (mitigated by service) or as rebound effect 3]. The aim of Software Engineering for Sustainability (SE4S) is to make use of methods and tools in order to achieve this notion of sustainable software.

Motivation There is a plethora of (new) journals, conferences and workshops where the topic pops up, so it is hard to get a comprehensive overview of the state of research. There is only one earlier systematic mapping study on sustainability in software engineering, namely the study performed by a subset of the authors of the work at hand from 2012. This first review [4] is now extended and analyzed in more depth and detail, as the first study did not differentiate research facets and knowledge areas. Furthermore, the first study revealed that only very few topics were in the actual area of 
software engineering, which is why the study then included related research on sustainable software systems outside of software engineering. As the topic has been researched very actively in the past few years, this second study leads to a larger set of data points that allow to draw more conclusions.

Research Objective Our aim is to provide an overview of the current state of research on software engineering for sustainability. The first step was our previous work with an earlier study on the available research [4], and now a related effort is made after only two years because the field has substantially evolved since then.

Contribution We contribute a systematic mapping study that follows the guidelines in 5. It takes into account the lessons learned from the previous study [4] by defining more robust research questions, using an adapted search string, and including a number of publication channels (journals, conferences and workshops) on the topic that have either been just recently established or were not indexed yet in the earlier study.

\section{STUDY DESIGN}

We describe the study design in terms of research questions, set-up, and conducted procedures.

\section{Research Questions (Scope).}

The overall research objective of the study is to give an overview of the current state of the art in supporting sustainability in software engineering research and practice. This is detailed in the following research questions:

RQ1 What research topics are being addressed?

RQ2 How have these research topics evolved over time?

RQ3 How is sustainability support performed (e.g., models and methods)?

RQ4 Which of those models and methods are used in practice?

RQ5 Which research type facets have been considered in the contributions?

RQ6 Which application domains have been considered?

RQ7 Which research groups are most active and what is the distribution between academics and practitioners?

\section{Roles \& Responsibilities.}

The roles and responsibilities for this project are defined in Tab. 1. We have two principal researchers (Birgit Penzenstadler and Ankita Raturi), three supporting researchers (Henning Femmer, Coral Calero, Xavier Franch), one internal reviewer (Debra Richardson) and two external reviewers (Daniel Méndez Férnandez and Marcela Genero).

\section{Search Strategy.}

Information Sources. The search process for this study is based on an automated search of the following indexing systems and digital libraries: DBLP, Science Direct, Web Of Science, INSPEC, IEEE Xplore, Springer, ACM, JSTOR, arXiv, Wiley, and Citeseer. Furthermore, we added manual searches on conference and workshop proceedings of 2013, as pretests of the search string have revealed that they did not show up in the search results of the indexing systems: ICT4S'13, GREENS'13, and RE4SuSy'13. The reason for them not being indexed was that it was still too early after their publication, but as we knew of their existence and rel-
Table 1: Roles and Responsibilities

\begin{tabular}{l|c|c|c|c|c|c|c|c} 
& $\mathrm{BP}$ & $\mathrm{AR}$ & $\mathrm{DR}$ & $\mathrm{CC}$ & $\mathrm{HF}$ & $\mathrm{XF}$ & $\mathrm{DM}$ & $\mathrm{MG}$ \\
\hline Develop protocol & $\mathrm{x}$ & & & & & & & \\
\hline Define search string & $\mathrm{x}$ & & $\mathrm{x}$ & & & & & \\
\hline Define classification scheme & $\mathrm{x}$ & & & & & & & \\
\hline Define data extraction form & $\mathrm{x}$ & & & & & & & \\
\hline Internal review of protocol & & & $\mathrm{x}$ & $\mathrm{x}$ & $\mathrm{x}$ & $\mathrm{x}$ & & \\
\hline External review of protocol & & & & & & & $\mathrm{x}$ & $\mathrm{x}$ \\
\hline Revise protocol & $\mathrm{x}$ & & & & & & & \\
\hline Identify primary research & $\mathrm{x}$ & $\mathrm{x}$ & & & & & & \\
\hline Retrieve primary research & $\mathrm{x}$ & $\mathrm{x}$ & & & & & & \\
\hline Clean from duplicates & & $\mathrm{x}$ & & & & & & \\
\hline Vote on search results & $\mathrm{x}$ & $\mathrm{x}$ & & $\mathrm{x}$ & $\mathrm{x}$ & $\mathrm{x}$ & & \\
\hline Assessment of voting & & & $\mathrm{x}$ & & & & & \\
\hline Data extraction & $\mathrm{x}$ & $\mathrm{x}$ & & & & & & \\
\hline Data synthesis & $\mathrm{x}$ & $\mathrm{x}$ & & & & & & \\
\hline Internal analysis validation & & & $\mathrm{x}$ & $\mathrm{x}$ & $\mathrm{x}$ & $\mathrm{x}$ & & \\
\hline External analysis validation & & & & & & & $\mathrm{x}$ & \\
\hline Complete technical report & $\mathrm{x}$ & $\mathrm{x}$ & & & & & & \\
\hline Write paper for EASE & $\mathrm{x}$ & $\mathrm{x}$ & & & & & & \\
\hline Review of report \& paper & & & $\mathrm{x}$ & $\mathrm{x}$ & $\mathrm{x}$ & $\mathrm{x}$ & $\mathrm{x}$ & \\
\hline
\end{tabular}

evance, we decided to include them in order to have more up-to-date results.

Search String. The aim for our search string is to capture all results that relate sustainability or environmental issues with software engineering or requirements for software systems. Not only in software engineering, but especially during the early phase of requirements engineering sustainability issues should emerge and be discussed, which is the reason for specifically including requirement in the search string. The search string ${ }^{1}$ used on all databases is:

(sustainab* OR ecolog* OR green) AND

(software engineering $\mathrm{OR}$ requirement* engineering $\mathrm{OR}$ requirement* specification $O R$ software specification $O R$ system specification)

We decided not to include "environment*" as alternative for sustainab*, ecolog* or green in the first parenthesis because pretests showed only false positives as it is a term frequently used for denoting the system context, operational context, or business context. The second parenthesis contains the part making it relevant for software engineering and the first parenthesis contains the part that links it to sustainability including synonyms and alternative terms that we know are in use. Although we explicitly list keywords in our search string that point to environmental sustainability, we are interested in all dimensions of sustainability as they are strongly related to each other.

Search Execution. We execute the search on the databases specified earlier. The search string is used to perform the search including the meta data fields title, abstract, and keywords. In case the search returned more than 100 results ordered according to the relevance with regard to the search string, we use the first 100 search results of each database. We retrieve the meta information (full citation and abstract) as well as the full texts. We consolidate the results and clean from duplicates. We provide the primary sources as well as a separate voting sheet per classification assessor in a Dropbox folder.

Study Selection Criteria. We chose the following inclusion criteria to select the relevant publications to answer our research questions: Relevance with respect to research questions, scientific soundness (see quality assessment in Sec. 2), and coverage of a software system (as opposed to pure hardware systems).

Exclusion Criteria are Environment used in the sense of sys-

\footnotetext{
${ }^{1}$ The search string used in the preceding study was (sustainab* OR environment* OR ecolog* OR green) AND (software engineering $O R$ requirement $O R$ software system)
} 
tem environment, not nature, and Ecosystem used as population of interacting systems, for example, agents.

\section{Study selection procedures.}

The process was conducted as follows: The five voters read all titles and abstracts and decide on the inclusion and exclusion for each entry according to the criteria given above. If unsure about an article, they read more of the paper until they are decided. Disagreements among voters are resolved by majority as we chose an uneven number of assessors. This also requires at least 3 out of 5 votes for decision taking. The internal reviewer reassesses the inclusion/exclusion of search results.

\section{Study quality assessment checklists and procedures.}

The following assessment checklist has been used to assess the quality of the studies under consideration: Peer-reviewed articles, reporting on background and context, description of research method, report on threats to validity.

For quality assessment, we performed internal and external reviews as also specified in Tab. 1. There were five internal reviews and three external ones. We conducted internal reviews of the protocol, of the voting, of the data extraction and classification, of the analysis and data synthesis, of the report. External reviews were performed of the protocol, of the analysis, of the report.

\section{Data extraction strategy.}

The principal researchers classify the studies according to the research type facets [7] and the knowledge area [8], as detailed in the list below. They extract information on topics, methods, frameworks, tools, case studies, and application domains.

The data extraction form captures the following data for each included primary resource:

- Metadata: Authors, Year of publication, Title, Source, Keywords, Research topic, Institution

- SWEBOK 8 knowledge area: Software Engineering Economics, Software Requirements, Software Testing, Software Construction, Software Configuration Management, Computing Foundations, Software Engineering Models and Methods, Software Maintenance, Mathematical Foundations, Software Design, Software Engineering Management, Software Engineering Professional Practice, Engineering Foundations, Software Engineering Process, or Software Quality.

- Research type facets 7]: Philosophical, Exploratory, Solution, Validation, Evaluation, Opinion, or Experience.

- Application domain (if applicable)

- Framework and/or Method (if applicable)

- Tool (if applicable)

\section{Synthesis of the extracted data.}

The principal researchers extract statistics and analyse the included results in further detail. They map out the current research. The internal reviewer assesses the analysis results and provides feedback. The external reviewers provide feedback. To conduct the data synthesis, we derived descriptive statistics for maps from the extracted data, performed semantic modeling of research topic clusters, mapped out current work, made timelines with amount of publica- tions according to research topic, research type facet, and knowledge area.

\section{RESULTS}

An overview of the search result numbers is provided in Tab. 2 The publications that were voted in by the majority of reviewers are listed later in Tab. 4. The 83 resulting publications were published quite across a range of journals, conferences, and workshops and covered a variety of topics, knowledge areas and research types. The full report is available online 6 .

Table 2: Overview of the search result numbers

\begin{tabular}{|l|l|}
\hline Total number of search results & 1278 \\
Total number after duplicate removal & 1039 \\
Voted in by at least one reviewer & 384 \\
Voted in by majority & 83 \\
\hline
\end{tabular}

\section{RQ1: What research topics are being addressed?.}

We used a variety of methods to structure and model the research topics of the 83 publications that were voted-in. Fig. 1 shows a simple weighted word cloud that was generated from the publication abstracts. It was created with Tagxedd ${ }^{2}$ which used a stemming algorithm to filter the textual input. The goal of this image was to gain a first impression of the topical content of the publications.

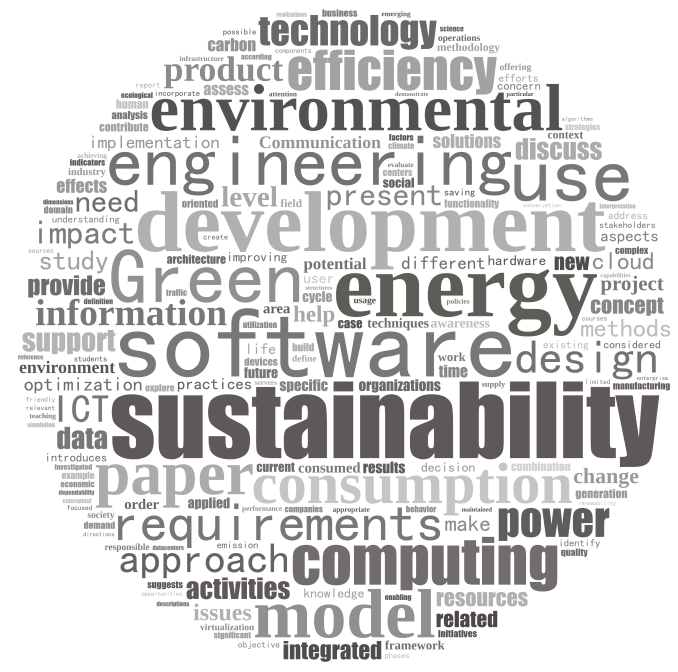

Figure 1: Weighted word cloud from the original abstracts of voted-in publications.

The next, more in depth analysis method used is called Topic modeling. This is a method for analyzing large data sets to elicit commonalities, in this case topics, which are clusters of words that frequently occur together in the data 9 . It is a "probabilistic model for uncovering the underlying semantic structure of a document collection" 10. We utilized the Machine Learning for LanguagE Toolkit (MALLET) ${ }^{3}$ that is popularly used for machine learning applications to text, including classification, clustering, natural language

\footnotetext{
www.tagxedo.com

3 http://mallet.cs.umass.edu
} 
processing, and topic modeling. The purpose of performing topic modeling on the dataset (consisting the abstracts of the voted-in publications) was to investigate what the 'hot topics' are in the domain of Software Engineering for Sustainability.

In order to be able to run the dataset through MALLET, we preprocessed the abstracts to be represented as a list of words associated with each publication. The dataset was also imported into MALLET using functionality that removed stop words and took into account basic word stemming. As our dataset of 83 documents was small, we only ran the trainer for 100 iterations. The goal was a qualitative corpus exploration 10, so we chose the top 10 topics for consideration. The modeling of the abstracts resulted in the topic clusters of the future of society, urban architecture and integration, energy efficiency, life cycle assessment, environmental management, smart grids, cloud services, carbon consumption, traffic strategies, and virtualization. Please refer to the complete protocol [6] for the full list of topic cluster keywords and further detailed analysis graphs.

Based on the word content of each abstract, and the output from the MALLET topic model, we were able to relate abstracts to the elicited topics. We pruned each topic down to 6 keywords that were most characteristic of the abstracts that belonged to each topic. Fig. 2 shows the resulting clusters of papers and the topics they belong to. The numbers in this graphic refer to the numbers in Tab. 4.

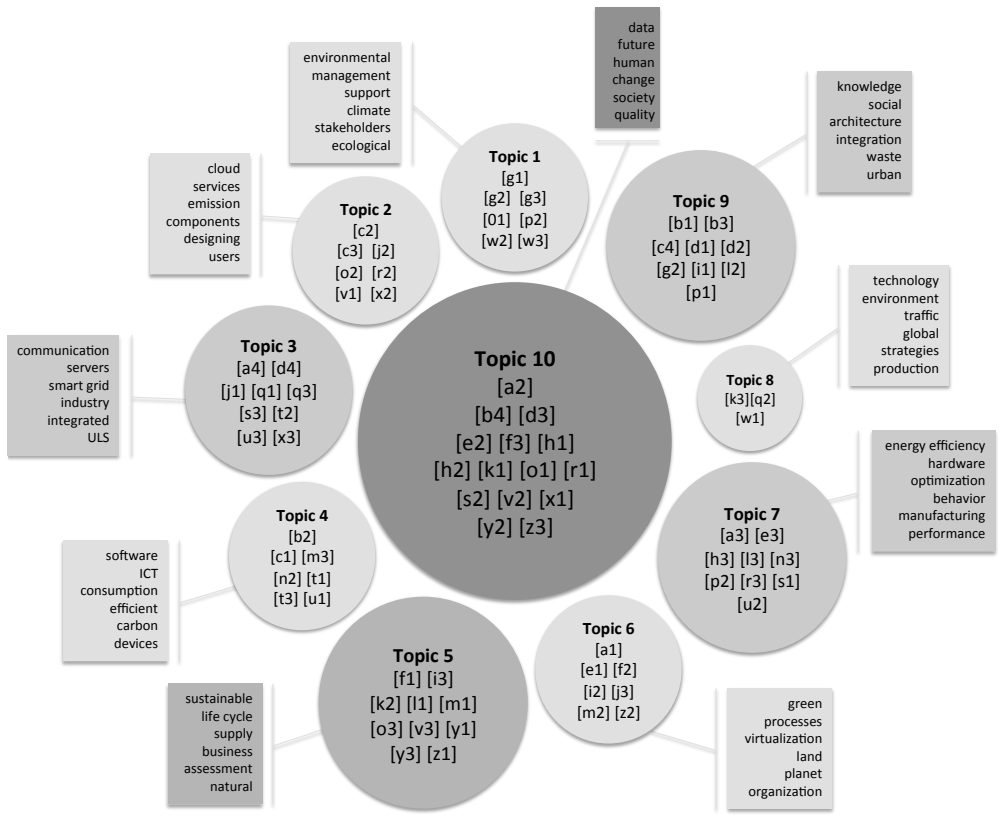

Figure 2: Topic Cluster Modeling of the Abstracts. Numbers in this graphic refer to the numbers in Tab. 4 .

During the data extraction phase of this study, we had classified each of the publications under a SWEBOK Knowledge Area 8, as well as under a Research Type Facet as described by 7]. In Fig . 3, we cross reference the Topic Clusters to the Knowledge areas and the Research Type Facets respectively, to allow for the identification of research hotspots.

Popular research in specific Knowledge Areas include: Software Engineering Process regarding Topic 10 [data, future, human, change, society, quality], and Software Design and Software Quality regarding Topic 5 [sustainable, life cycle, supply, business, assessment, natural]. Popular research using specific Research Type Facets include: Solutions research in Topic 3 [communication, servers, smart grid, industry, integrated, ULS], Topic 5 [sustainable, life cycle, supply, business, assessment, natural], Topic 7 [energy efficiency, hardware, optimization, behavior, manufacturing, performance], and Topic 10 [data, future, human, change, society, quality].

\section{RQ2: How have these research topics evolved over time?.}

The answer to RQ 2 needs a prelude on how the publications, and therefore our data points, are distributed over time. As depicted in Fig. 4 there were 40 new relevant publications in the last two years alone. A description of the evolution of the topics over time is somewhat limited, as this constitutes a majority of publications that are in domain of Software Engineering for Sustainability.

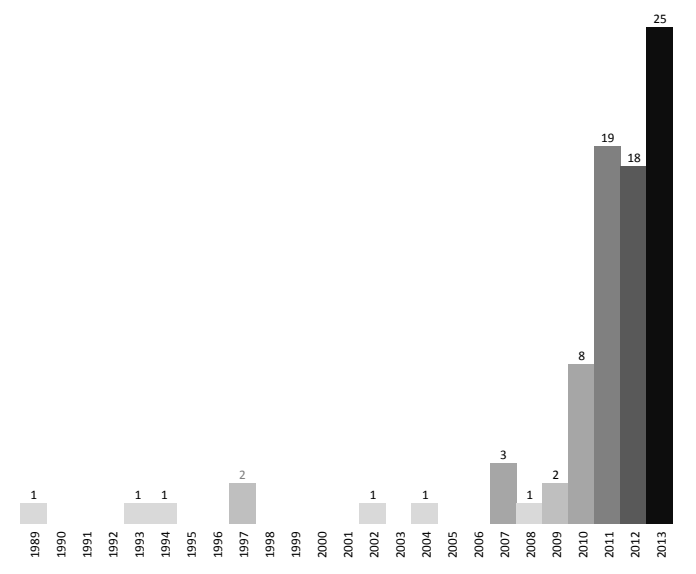

Figure 4: Distribution of the publications over time.

\section{RQ3: How is sustainability support performed?.}

There is a wide range of models, methods, frameworks, and tools that are proposed in the publications and used in research. They include standard software engineering support (like goal modeling and service modeling) as well as general purpose methods (like interviews and statistics) as well as more domain-specific methods from systems engineering (life cycle assessment), geosciences (global position system) and the energy domain (measuring devices).

- Software engineering methods \& tools: goal modeling [k1, e2, p3], stakeholder modeling [e2, w3], agent modeling [d1], service modeling [g2], process modeling [d2, $\mathrm{f} 2,12]$, simulation $[\mathrm{r} 3,11]$

- General purpose methods \& tools: interviews [t1], statistics [p2], surveys [u1]

- Systems Engineering: life cycle assessment [f1, l1]

- Geo Sciences: global position system, internet map services [m1, w1]

- Earth Sciences: environmental information systems [p1, q3]

- Urban Planning: simulation [i1, d1] 


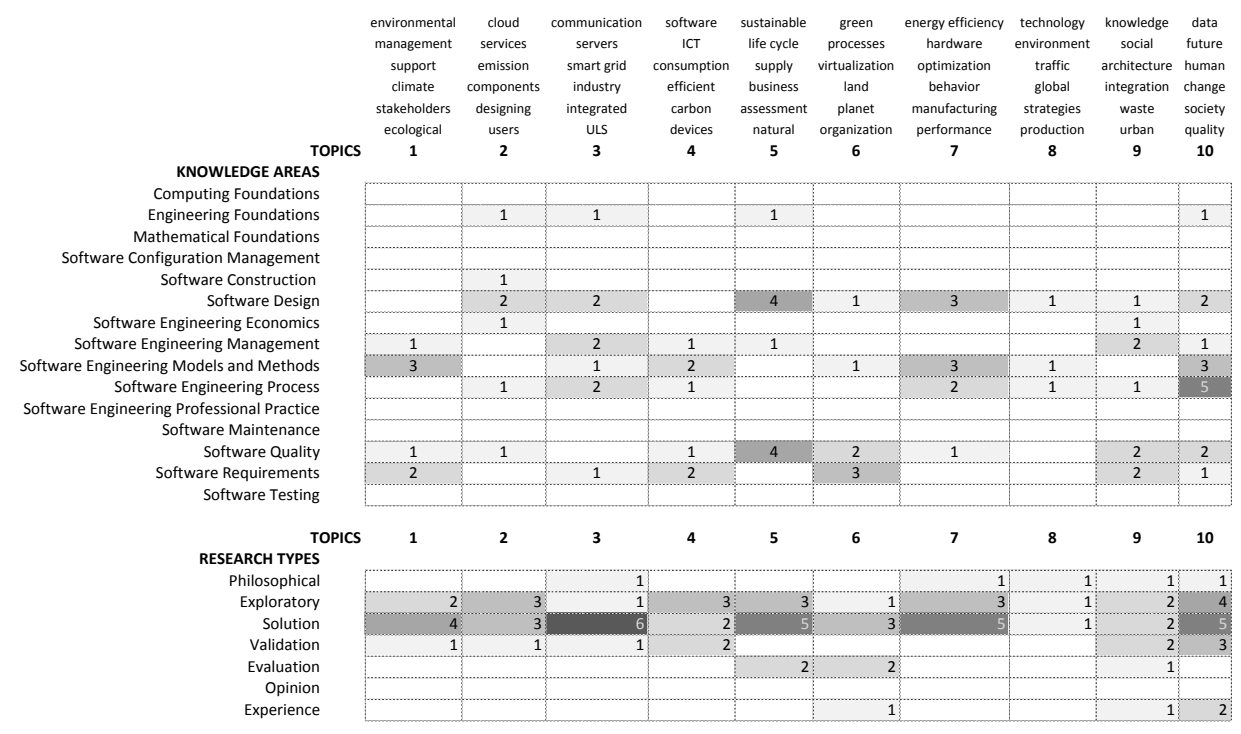

Figure 3: Top: Topic Clusters related to Knowledge Areas, Bottom: Topic Clusters related to Research Topics

- Energy Management: measuring devices [v2, k2, d3], traffic management systems [w1]

This plethora of used approaches only leads to the conclusion that there are many different roads being explored but there are no methods and models yet that can be considered as established for SE4S.

\section{RQ4: Which models and methods are used in prac- tice?.}

In order to report on which means are used in practice (as opposed to being only proposed as a solution in a publication), when considering Fig. 3 it is clear that there are not many publications of the research facet Evaluation or Experience. Evaluation papers are [o2, p2, a3, r3, d4], and Experience papers are [b1, c1, e2, s3]. Due to this low number, it does not make sense to draw further conclusions on the state of practice. It also leaves the question of whether the topic is not really triggering a state of practice at all or whether it is simply not published much on yet.

\section{RQ5: Which research methods have been considered in the contributions?.}

In Fig. 5, we display the relation of knowledge areas 8 to research facets 7.

As represented in Fig. 5, there are many contributions of the type Exploratory and Solution, but on the other hand none of the type Opinion and very few in Experience and Evaluation. This indicates a young and still somewhat immature research area which needs to perform more evaluation and encourage practitioners to report on experiences.

\section{RQ6: Which application domains have been consid-} ered?.

As not all publications are considering an explicit application domain, but more than $50 \%$ have a generic approach across application domains, we classified papers either according to an application domain or a focus domain to be able to differentiate them in categories. As opposed to the
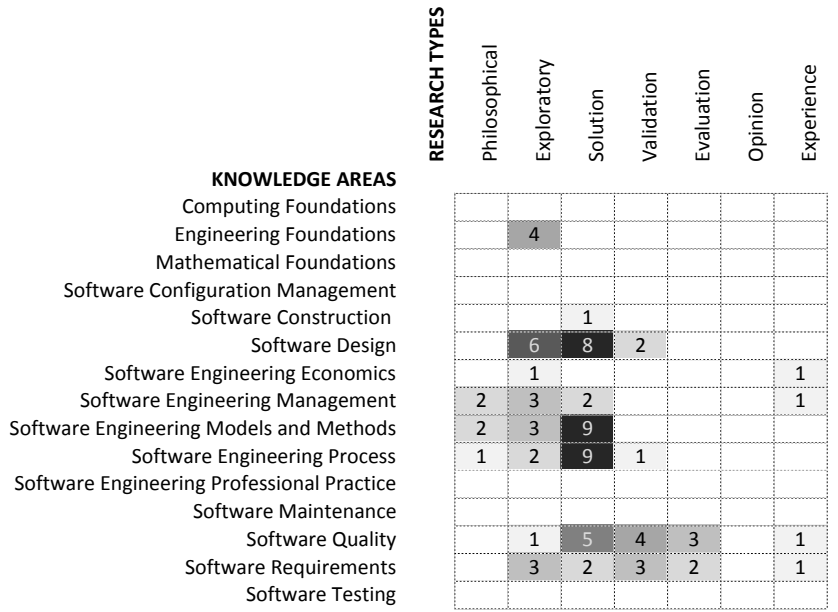

Figure 5: Correlation of Knowledge Areas to Research Facets.

topic clusters discussed in RQ 1, these domains were not extracted automatically, but assigned manually by the researchers. Furthermore, a subsequent mapping between the automatically extracted research topic clusters and the application domains did not lead to significant correlation, thereby undermining the fact that they are worth distinguishing. We found ten such domains:

- Software Engineering \& Lifecycle: Publications that do not refer to a specific application domain but presented generic approaches related to software engineering and the software lifecycle.

- Energy Efficiency: Publications that dedicate their work specifically to energy efficiency topics.

- Services, Mobile \& Cloud: Publications that research topics in a service-oriented paradigm, often including mobile aspects and/or cloud computing, including research that monitors and improves traffic in cloud computing. 
Table 3: Number of Voted-in papers according to Application and Focus Domain

\begin{tabular}{|l|l|}
\hline Application / Focus Domain & Publications \\
\hline Software Engineering \& Lifecycle & 22 \\
Energy Efficiency & 5 \\
Services, Mobile \& Cloud & 10 \\
Business \& Economics & 5 \\
Systems Engineering \& ICT & 12 \\
ULS Green Computing & 7 \\
Mechanics \& Manufacturing & 3 \\
Nature \& Agriculture & 5 \\
Metropolitan Areas \& Housing & 9 \\
Software Engineering Education & 5 \\
\hline
\end{tabular}

- Business \& Economics: This focus domain includes publications on business processes and organizational issues as well as globalization.

- Systems Engineering \& ICT: Many contributions go over the boundaries of software, but consider ICT and whole systems, leading to a broader application of the principles of sustainability.

- ULS Green Computing: (Ultra) Large-scale systems have become a focus in computing as optimization of software on that level can potentially have a big impact on the overall resource consumption of ICT.

- Mechanics \& Manufacturing: Few contributions specifically address green (re-)manufacturing.

- Nature \& Agriculture: This focus domain entails developing systems for supporting sustainability in agriculture as well as improving environmental modeling for monitoring nature and gaining insights on related data points and clusters (e.g., on climate change).

- Metropolitan Areas \& Housing: A number of approaches targets urban management, including traffic, transportation, smart homes, and urban ecosystems.

- Software Engineering Education: Last but not least, five publications address how to incorporate the topic of sustainability into software engineering education.

The application domains and focus areas that have been considered in the publications are listed in Tab. 3. The publications are all referenced and clustered according to these domains in Tab. 4.

Figure 6 briefly summarizes the mapping of the manual classification of publications to Application Domains to the resultant topic cluster modeling classification of publications to the Topic Clusters. This figure shows the distribution of topics that occur in each of the application domains of the SE4S publications. Fig. 6 shows that the research topic clusters on the future of society, urban architecture and integration, energy efficiency, life cycle assessment, environmental management, smart grids, cloud services, carbon consumption, traffic strategies, and virtualization (as in Fig. 2) do not significantly correlate with the distribution of the application domains. However, alignments are perceivable for a small subset.

RQ7: Which research groups are most active in researching the topic and what is the distribution between academics and practitioners?

The network graph was constructed based on the authors of the 83 voted-in publications. It was generated using Many Eye: $4^{4}$ an experimental Visualization web service by IBM

${ }^{4}$ http://www-958.ibm.com/software/analytics/ manyeyes/
Research.

Apart from that there are a 197 unique authors, but as was shown in Fig. 4 most have been active in the last 3 years. We found 56 connected subgraphs (some of which were single author nodes), three of which are major research clusters, where authorship spans more than one or two papers. These are also fairly globally distributed, with even some intercontinental collaborations. These three interesting subgraphs are shown in Fig. 7 (a larger version can be found in [6]). The distribution of publications between academia and industry is currently unbalanced with roughly $80 \%$ of reported evidence coming from academia, the rest being distributed between industry and mixed collaborations. This distribution was derived from the affiliation that the authors provided for the publication.

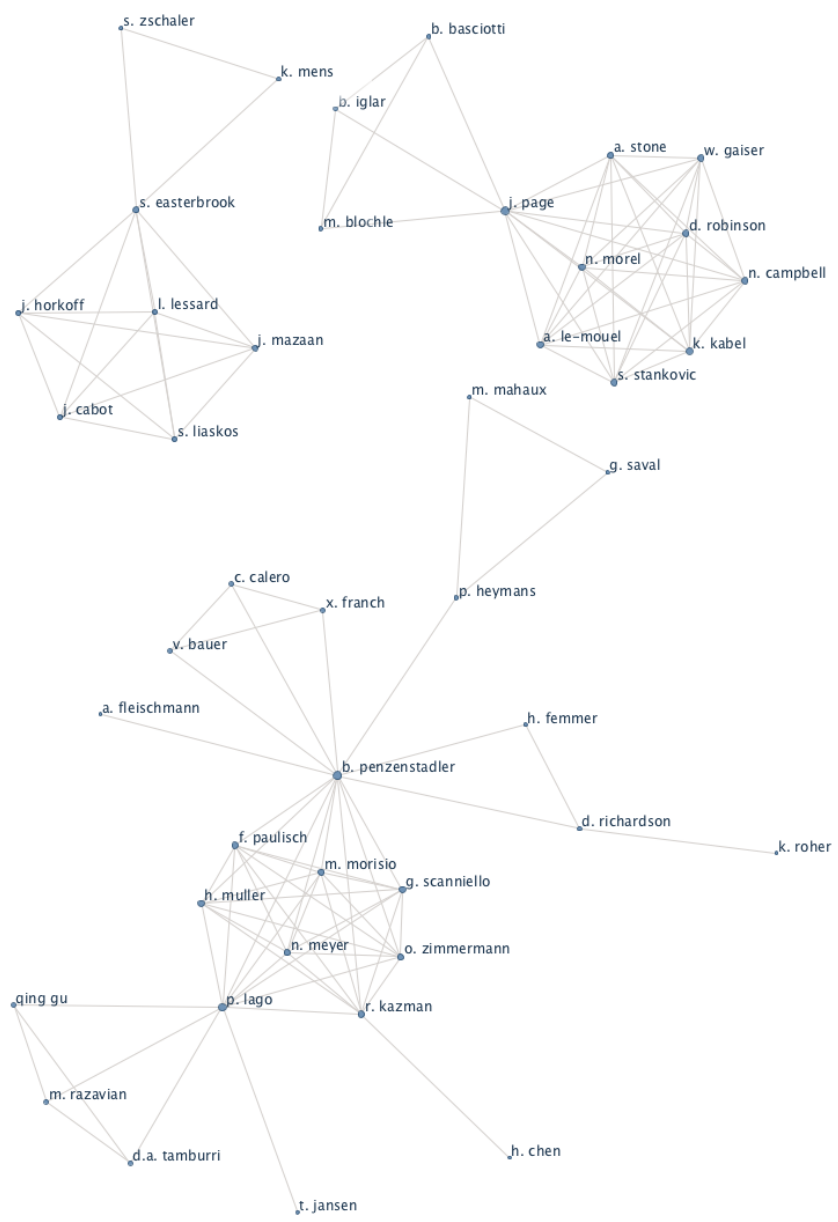

Figure 7: Three largest author network subgraphs in detail; a larger version can be found in 6

\section{DISCUSSION \& THREATS}

This section provides a discussion of the results and of the threats to validity for this study.

\section{Completeness of Results.}

During the voting period, there were suggestions by reviewers for other papers they knew of, which they had expected to show up in the results but did not, were carefully checked by the principal researchers. 


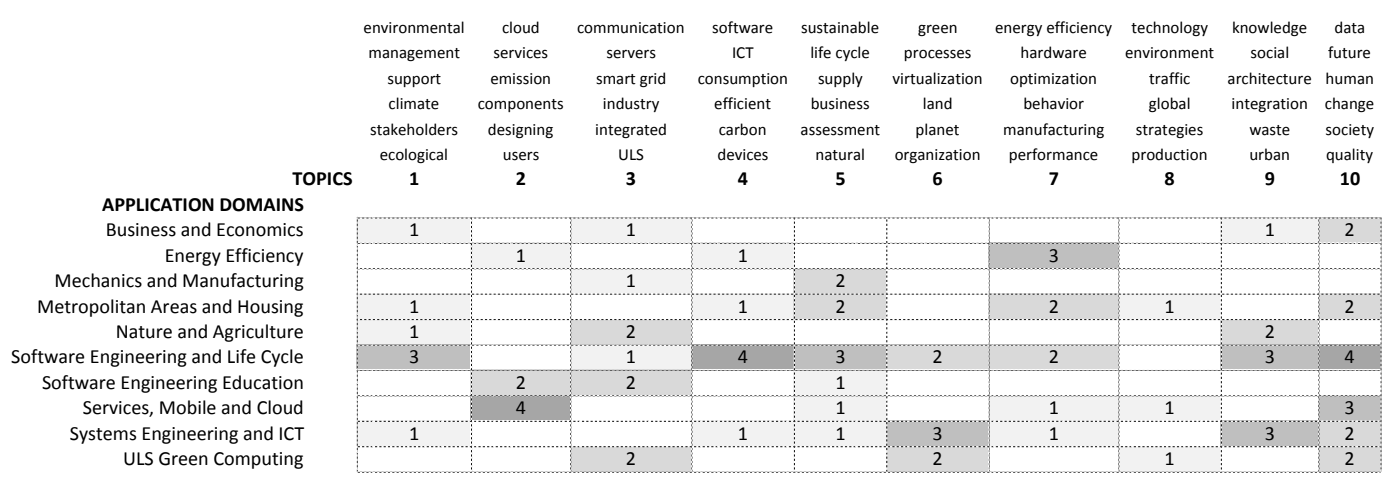

Figure 6: Correlation of Application Domains to Topic classification

One reason for why some of the expected results had not shown up in the automatic search results was that they had not applied to the first part of the search string. The first part (sustainab* OR ecolog* OR green) required an explicit link of the research to sustainability concerns. This was not the case for many energy efficiency publications, therefore these may be underrepresented in the results of our study.

Another reason for missing expected results was that papers did not match the second part of the search query (software engineering $O R$ requirement* engineering $O R$ requirement* specification OR software specification OR system specification). We encountered a few papers, for example, from the GREENS workshop at ICSE 2013, that we consider relevant to the research area, but did not show up in the results because they used other terms like 'software quality' to classify their research. We conclude that some software engineering researchers who work in the analyzed area of investigation are missing from the results because they used more specific terms and did not include the more general terms 'software engineering', or 'software specification', for example in energy efficiency and software quality.

\section{Search Engine Correctness.}

Each of the information sources (i.e. the indexing systems and digital libraries listed in 2 evaluated boolean search queries according to their own mechanism. Therefore, when an information source did specify query rules, the search string was adapted accordingly. An issue that was prevalent in some information sources was that there were different search results for semantically equivalent queries based on the order of operations. To this extent, we can not guarantee for the quality of the automatically executed queries in those information sources. However, as we used a wide range of search engines, we hope we have mitigated that effect as far as possible.

\section{Manual Additions.}

We have manually added a small set of proceedings of venues that are very relevant to the research area to the set of automatically retrieved papers due to the fact that the newest (2013) edition of these conferences and workshops was not yet indexed by the search engines. We did this in order to make the selection pool for relevant papers as up-to-date as possible. In our understanding, this does not introduce a strong bias for the research but rather merely offers a potential qualitative improvement of the results.

\section{Data Synthesis.}

The data synthesis was performed partially automatic, partially manually. For the automatic part, we relied on topic cluster modeling and generated graphics to aggregate information in a form that is more easily perceived by human cognition that pure numbers. These tools have been used widely throughout this and other research communities and we trust they are reliable and produce valid results. For the manual part, i.e. the classification according to knowledge areas, research facets, and focus domains, we performed the data synthesis to the best of our knowledge. However, human judgement is always subjective to a certain degree, so other researchers might have chosen slightly different terms for application domains or keywords for methods and frameworks. This threat was mitigated by internal and external reviews.

\section{CONCLUSIONS}

This paper presented a systematic mapping study to provide an overview of the current body of knowledge and research for software engineering for sustainability. This objective was detailed in seven research questions (RQ 1-7) on research topics, methods \& tools, and application domains. The work was carried out by two principal researchers, three supporting researchers, an internal reviewer and two external reviewers over the course of 4 months. Please refer to the full report for additional information 6 .

The topic of SE4S has received wide-spread attention in the software engineering community over the past few years. Due to the fact of being a relatively new area of research, there is rather little reported evidence of establishment in practice. At the same time, industry has recognized the topic and use the term sustainability all over, reminding of the Green IT hype, but now broadened to sustainability. As Green IT practices are by now further established in practice, hope remains that the same will come true for other sustainability practices. The low number of evaluation and experience papers in the reported evidence also suggest that the research area including its solutions are still somewhat immature. Furthermore, the fair distribution over a range of journals and venues indicates that the research community is still forming. However, the large number of topic clusters, focus areas and application domains indicates that research is being conducted in broad coverage of the area of SE4S. The following list sums up the major conclusions from the reported evidence. 
RQ1 The research topic clusters that have been addressed include a variety of aspects ranging across the future of society, urban architecture and integration, energy efficiency, life cycle assessment, environmental management, smart grids, cloud services, carbon consumption, traffic strategies, and virtualization. The majority of publications are in the knowledge areas of Software Design, Engineering Management, Models and Methods, Process, Quality, and Requirements.

RQ2 Evolution of the research topics over time reveals a strong general development over the last four years, especially in the topic clusters of future of society, life cycle assessment, and energy efficiency.

RQ3 Sustainability support is performed by a variety of models and methods that include general purpose (interviews, statistics, surveys), software engineering (goals, stakeholders, services, processes), systems engineering (LCA), as well as methods from geo sciences, earth sciences, urban planning, and energy management.

RQ4 The usage of the approaches in practice is very limited in the reported evidence.

RQ5 The most prominent research type facets were Exploratory and Solution.

RQ6 The application domains that were predominantly considered are Software Engineering and Lifecycle, Systems Engineering and ICT, Energy Efficiency, Mobile Services and Cloud, Business and Economics, ULS Computing, Mechanics and Manufacturing, Nature and Agriculture, Metropolitan Areas and Housing, and Software Engineering Education.

RQ7 There are three rather active research groups but research is performed all over the world and distribution between academia and industry is currently unbalanced with roughly $80 \%$ of reported evidence from academia, the rest distributed between industry and mixed collaborations.

The aggregation of results and overviews in graphics and tables as well as the compact table of included publications may be considered as a compact overview of the field of Software Engineering for Sustainability.

Due to the facts that SE4S has significantly gained importance over the past few years and that it has been intensely researched by a world-wide community, we conclude that there is need for a future roadmap that identifies the major research gaps and outlines promising options of how to fill these gaps.

Acknowledgements. We would like to thank Daniel Méndez Férnandez and Marcela Genero for serving as external reviewers and for helpful feedback. This work is part of the DFG EnviroSiSE project (grant number PE2044/1-1).

\section{REFERENCES}

[1] L. Hilty, W. Lohmann, and E. Huang, "Sustainability and ICT - an overview of the field," in Proceedings of the EnviroInfo 2011, 2011.

[2] B. Brown, M. Hanson, D. Liverman, and R. Merideth, "Global sustainability: Toward definition," EnvironmentalManagement, vol. 11, no. 6, pp. pp. 713-719, 1987.

[3] L. Hilty et al., "The relevance of information and communication technologies for environmental sustainability," Environm. Modelling $\& 5$ Software, vol. 21, no. 11, pp. 1618 - 1629, 2006.

[4] B. Penzenstadler, V. Bauer, C. Calero, and X. Franch, "Sustainability in software engineering: A systematic literature review," in International Conference on Evaluation and Assessment in Software Engineering (EASE), 2012.

[5] B. Kitchenham and S. Charters, "Guidelines for performing systematic literature reviews in software engineering," tech. rep., Software Engineering Group, Keele University, 2007.

[6] B. Penzenstadler, A. Raturi, D. Richardson, C. Calero, H. Femmer, and X. Franch, "Systematic mapping study on software engineering for sustainability (se4s) - protocol and results." http: //ics.uci.edu/ `bpenzens/pdfs/SMSprot14.pdf 2014.

[7] R. Wieringa, N. Maiden, N. Mead, and C. Rolland, "Requirements engineering paper classification and evaluation criteria: a proposal and a discussion," Requir. Eng., vol. 11, pp. 102-107, Dec. 2005.

[8] IEEE, "Software engineering body of knowledge (swebok)," 2013. http://www.swebok.org.

[9] T. L. Griffiths and M. Steyvers, "Finding scientific topics," PNAS, vol. 101, no. 1, pp. 5228-5235, 2004.

[10] D. M. Blei and J. D. Lafferty, Text Mining: Classification, Clustering, and Applications, ch. Topic Models, pp. 71-94. Chapman and Hall, 2009. 
Table 4: Voted-in papers according to Application Domain

\begin{tabular}{|c|c|c|c|c|}
\hline Ref & Year & Author & Title & Output Channel \\
\hline \multicolumn{5}{|c|}{ Application / Focus Domain: General Software Engineering \& Lifecycle } \\
\hline [a1] & 1989 & Cohill & The human factors design process in software development & 3rd Intl. Conf. HCI \\
\hline [z1] & 2011 & Johann et al. & $\begin{array}{l}\text { Sustainable development, sustainable software, and sustainable software } \\
\text { engineering: An integrated approach }\end{array}$ & $\begin{array}{l}\text { IC Humanities, Science Engi- } \\
\text { neering Research }\end{array}$ \\
\hline$[\mathrm{d} 2]$ & 2011 & $\begin{array}{l}\text { Maharmeh, } \\
\text { Saeed }\end{array}$ & $\begin{array}{l}\text { Application of a composite process framework for managing green ICT } \\
\text { applications development }\end{array}$ & $\begin{array}{l}\text { Handbook of Research on Green } \\
\text { ICT }\end{array}$ \\
\hline$[\mathrm{f} 2]$ & 2011 & Naumann et al. & $\begin{array}{l}\text { The GREENSOFT Model: A reference model for green and sustainable } \\
\text { software and its engineering }\end{array}$ & $\begin{array}{l}\text { Sustainable Computing: Infor- } \\
\text { matics and Systems }\end{array}$ \\
\hline$[12]$ & 2011 & Shenoy, Eeratta & $\begin{array}{l}\text { Green software development model: An approach towards sustainable soft- } \\
\text { ware development }\end{array}$ & IEEE India Conference \\
\hline$[\mathrm{n} 2]$ & 2012 & Agarwal et al. & Sustainable approaches and good practices in green software engineering & $\begin{array}{l}\mathrm{J} \text { of Research and Reviews in } \\
\text { Computer Science }\end{array}$ \\
\hline [t2] & 2012 & Hindle & Green mining: Investigating power consumption across versions & ICSE \\
\hline [w2] & 2012 & Johann et al. & $\begin{array}{l}\text { How to measure energy-efficiency of software: Metrics and measurement } \\
\text { results }\end{array}$ & W GREENS \\
\hline [y2] & 2012 & Lami, Buglioni & Measuring Software Sustainability From a Process-Centric Perspective & W on Software Measurement \\
\hline [a3] & 2012 & $\begin{array}{l}\text { Noureddine et } \\
\text { al. }\end{array}$ & A preliminary study of the impact of software engineering on GreenIT & W GREENS \\
\hline [b3] & 2012 & $\begin{array}{l}\text { Penzenstadler et } \\
\text { al. }\end{array}$ & Sustainability in software engineering: A systematic literature review & IC EASE \\
\hline [e3] & 2012 & Schubert et al. & Profiling Software for Energy Consumption & C GreenCom \\
\hline [f3] & 2013 & Lago et al. & Exploring initial challenges for green software engineering & SIGSOFT SE Notes \\
\hline [m3] & 2013 & Dick et al. & Green software engineering with agile methods & GREENS \\
\hline$[\mathrm{t} 3]$ & 2013 & Kern et al. & $\begin{array}{l}\text { Green Software and Green Software Engineering - Definitions, Measure- } \\
\text { ments, and Quality Aspects }\end{array}$ & ICT4S \\
\hline [v3] & 2013 & Naumann & Classifying Green Software Engineering - The GREENSOFT Model. & J Software-Technik Trends \\
\hline [w3] & 2013 & $\begin{array}{l}\text { Penzenstadler et } \\
\text { al. }\end{array}$ & Who is the advocate? Stakeholders for sustainability & W GREENS \\
\hline$[\mathrm{x} 3]$ & 2013 & $\begin{array}{l}\text { Roher, Richard- } \\
\text { son }\end{array}$ & $\begin{array}{l}\text { A proposed recommender system for eliciting software sustainability re- } \\
\text { quirements }\end{array}$ & W USER \\
\hline [y3] & 2013 & Kocak et al. & $\begin{array}{l}\text { The Impact of Improving Software Functionality on Environmental Sus- } \\
\text { tainability }\end{array}$ & ICT4S \\
\hline [z3] & 2013 & $\begin{array}{l}\text { Roher, Richard- } \\
\text { son }\end{array}$ & Sustainability requirement patterns & W RePa \\
\hline [a4] & 2013 & Sventinovic & Strategic requirements engineering for complex sustainable systems & J Systems Engineering \\
\hline [b4] & 2013 & Penzenstadler & Towards a definition of sustainability in and for software engineering & $\mathrm{SAC}$ \\
\hline \multicolumn{5}{|c|}{ Application / Focus Domain: Energy Efficiency } \\
\hline [b2] & 2011 & Kutsuki & $\begin{array}{l}\text { Developing and Providing Software that Helps to Reduce Environmental } \\
\text { Burden }\end{array}$ & Fujitsu Journal \\
\hline [p2] & 2012 & Capra et al. & $\begin{array}{l}\text { Is software green? Application development environments and energy ef- } \\
\text { ficiency in open source applications }\end{array}$ & $\begin{array}{l}\text { Information and Software Tech- } \\
\text { nology }\end{array}$ \\
\hline [s2] & 2012 & Gotz et al. & Approximating quality contracts for energy auto-tuning software & W GREENS \\
\hline [n3] & 2013 & $\begin{array}{l}\text { Grosskop, } \\
\text { Visser }\end{array}$ & Energy Efficiency Optimization of Application Software & $\begin{array}{l}\text { B Green and Sustainable Com- } \\
\text { puting }\end{array}$ \\
\hline [r3] & 2013 & $\begin{array}{l}\text { Grosskop, } \\
\text { Visser }\end{array}$ & Identification of Application-Level Energy-Optimizations & ICT4S \\
\hline \multicolumn{5}{|c|}{ Application / Focus Domain: Services, Mobile \& Cloud } \\
\hline [m1] & 2010 & Ager et al. & $\begin{array}{l}\text { Internet map services: new portal for global ecological monitoring, or } \\
\text { geodata junkyard? }\end{array}$ & $\begin{array}{l}\text { IC on Computing for Geospatial } \\
\text { Research \& Application }\end{array}$ \\
\hline$[\mathrm{ol}]$ & 2010 & Bahsoon & $\begin{array}{l}\text { A Framework for Dynamic Self-optimization of Power and Dependability } \\
\text { Requirements in Green Cloud Architectures }\end{array}$ & $\begin{array}{l}\text { Europ. Conf. on Software Archi- } \\
\text { tecture }\end{array}$ \\
\hline [u1] & 2011 & Amsel et al. & Toward sustainable software engineering & IC on Software Engineering \\
\hline [v1] & 2011 & Atkinson et al. & $\begin{array}{l}\text { Modelling as a Service (MaaS): Minimizing the Environmental Impact of } \\
\text { Computing Services }\end{array}$ & W GREENS \\
\hline [c2] & 2011 & Lago, Jansen & $\begin{array}{l}\text { Creating Environmental Awareness in Service Oriented Software Engineer- } \\
\text { ing }\end{array}$ & W Service-Or. Computing \\
\hline$[\mathrm{j} 2]$ & 2011 & Sasikala & $\begin{array}{l}\text { Architectural strategies for green cloud computing: environments, infras- } \\
\text { tructure and resources }\end{array}$ & $\begin{array}{l}\text { J on Cloud Applications and } \\
\text { Computing }\end{array}$ \\
\hline [c3] & 2012 & Razavian et al. & $\begin{array}{l}\text { Modeling to support communication and engineering of service-oriented } \\
\text { software }\end{array}$ & $\begin{array}{l}\text { W Software Services and Sys- } \\
\text { tems Research }\end{array}$ \\
\hline [g4] & 2013 & $\begin{array}{l}\text { Atkinson, } \\
\text { Schulze }\end{array}$ & Towards application-specific impact specifications and GreenSLAs & W GREENS \\
\hline [k3] & 2013 & $\begin{array}{l}\text { Chauhan, Sax- } \\
\text { ena }\end{array}$ & A Green Software Development Life Cycle for Cloud Computing & J IT Professional \\
\hline [13] & 2013 & Corral et al. & A method for characterizing energy consumption in Android smartphones & GREENS \\
\hline \multicolumn{5}{|c|}{ Application Domain: Business \& Economics } \\
\hline [c1] & 1994 & Jones & Globalisation of software supply and demand & J Software Engineering \\
\hline [g2] & 2007 & Ramakrishnan & $\begin{array}{l}\text { Business process ontology and software service models for environmentally } \\
\text { sustainable manufacturing enterprises }\end{array}$ & IC ITI \\
\hline$[\mathrm{k} 1]$ & 2009 & Cabot et al. & $\begin{array}{l}\text { Integrating sustainability in decision-making processes: A modelling strat- } \\
\text { egy }\end{array}$ & $\begin{array}{l}\text { Intl Conf on Software Engineer- } \\
\text { ing }\end{array}$ \\
\hline [y1] & 2011 & $\begin{array}{l}\text { Harmon, } \\
\text { Demirkan }\end{array}$ & $\begin{array}{l}\text { The corporate sustainability dimensions of service-oriented information } \\
\text { technology }\end{array}$ & SRII Global Conference \\
\hline$[\mathrm{e} 2]$ & 2011 & Mauhaux et al. & Discovering Sustainability Requirements: An Experience Report & $\begin{array}{l}\text { WC Requirements Engineering: } \\
\text { Foundations for Software Quality }\end{array}$ \\
\hline
\end{tabular}




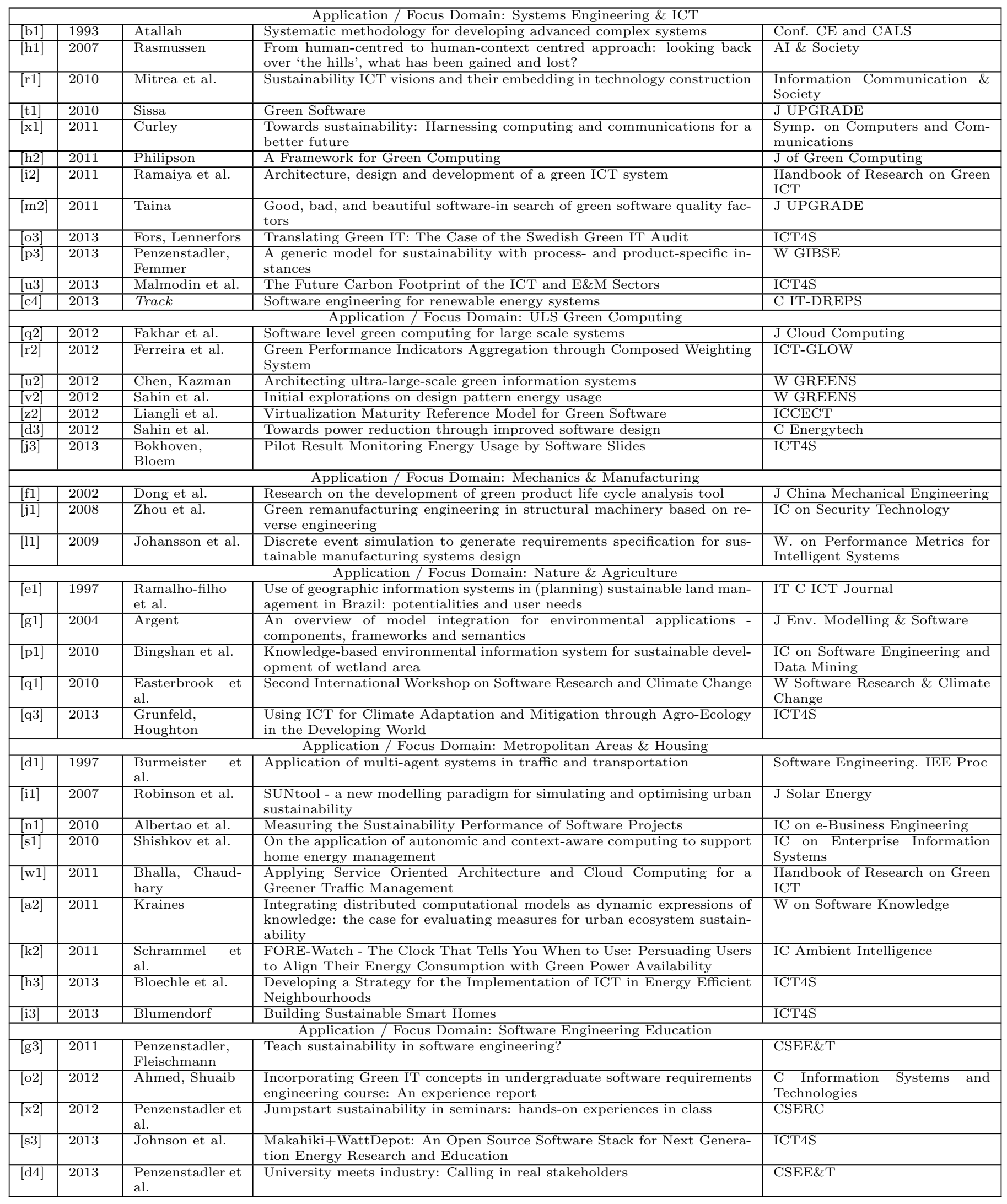

